\title{
Multi-scale analysis of the spatial structure of China's major function zoning
}

\author{
WANG Yafei ${ }^{1,2},{ }^{*}$ FAN Jie ${ }^{1,2}$ \\ 1. Key Laboratory of Regional Sustainable Development Modeling, Institute of Geographic Sciences and Nat- \\ ural Resources Research, CAS, Beijing 100101, China; \\ 2. College of Resources and Environment, University of Chinese Academy of Sciences, Beijing 100049, China
}

\begin{abstract}
The spatial structures of China's Major Function Zoning are important constraining indicators in all types of spatial planning and key parameters for accurately downscaling major functions. Taking the proportion of urbanization zones, agricultural development zones and ecological security zones as the basic parameter, this paper explores the spatial structures of major function zoning at different scales using spatial statistics, spatial modeling and landscape metrics methods. The results show: First, major function zones have spatial gradient structures, which are prominently represented by latitudinal and longitudinal gradients, a coastal distance gradient, and an eastern-central-western gradient. Second, the pole-axis system structure and core-periphery structure exist at provincial scales. The general principle of the pole-axis structure is that as one moves along the distance axis, the proportion of urbanization zones decreases and the proportion of ecological security zones increases. This also means that the proportion of different function zones has a ring-shaped spatial differentiation principle with distance from the core. Third, there is a spatial mosaic structure at the city and county scale. This spatial mosaic structure has features of both spatial heterogeneity, such as agglomeration and dispersion, as well as of mutual, adjacent topological correlation and spatial proximity. The results of this study contribute to scientific knowledge on major function zones and the principles of spatial organization, and it acts as an important reference for China's integrated geographical zoning.
\end{abstract}

Keywords: China; major function zoning; multi-scale; spatial gradient; pole-axis; core-periphery; spatial mosaic

\section{Introduction}

There are two concurrent major systems that affect the Earth's surface: the physical geography system and the socio-economic system (Fan et al., 2017). Every area has multiple function attributes, such as an ecological service function, human production function or living

Received: 2019-05-10 Accepted: 2019-07-20

Foundation: National Natural Science Foundation of China, No.41630644; Innovative Think-tank Foundation for Young Scientists of China Association for Science and Technology, No.DXB-ZKQN-2017-048

Author: Wang Yafei (1988-), PhD, specialized in spatial modeling and regional sustainability science.

E-mail: wangyafei@igsnrr.ac.cn

"Corresponding author: Fan Jie (1961-), PhD and Professor, E-mail: fanj@igsnrr.ac.cn 
function ( $\mathrm{Fu}, 2017$; Fan et al., 2018). The essential difference between areas lies in the importance and vulnerability of their ecosystems as well as attitudes toward human production and lifestyles (Fan and Li, 2009; Russell et al., 2013). Studying the relationship between people and land to construct a sustainable geographical or landscape pattern is currently an important frontier in sustainability science (Liu et al., 2007; Kates, 2011; Wu, 2013; Baerwald et al., 2016). A sustainable geographic or landscape pattern should offer both a sustainable supply of ecosystem service functions and orderly socio-economic spatial organization, and a necessary prerequisite is determining the regional function of each portion of land (Loorbach et al., 2017; Fan et al., 2019). A regional function is the overall role played by a certain region within a larger geographical area in terms of its natural resource and eco-environmental systems as well as its production and living activities. A regional function structure is a spatial structure determined by surface functions that solves the long-standing issue of calculating quantitative values in spatial management by constraining the functions, scale and proportional relationships involved in regional development. Like an industrial structure, this spatial structure characterized by a combinational relationship between spaces with regional functions also produces or affects economic, social and ecological benefits, with effects that are long lasting (Wang and Fan, 2019a). Disrupting the rational proportional relationship between regional function spaces or going beyond the upper development limit of a region can be highly detrimental to development due to the resulting disorderly spatial structure. Regional function structures differ, however, depending on differences in the geographic environment, development levels and development modes; they also change at different spatial scales. Determining a reasonable proportional relationship for surface spaces as well as specific quantitative structural features and evolution principles are topics that require further exploration.

Previous research on regional function structures has tended to focus on land cover or land use structures, land multi-functionality, landscape multi-functionality, and the relationship between production, living and ecological spaces. In research on land cover or land use structures, scholars from various countries have used multi-temporal and multi-resolution satellite remote sensing images to conduct relevant studies (Esmail et al., 2019; Mohammed et al., 2019), or evolutionary characteristics have been analyzed based on type, structure and rate of change of land cover/utilization in order to explore correlations between different types of time nodes and areas (Li et al., 2016; Qiao et al., 2018), which is then used to characterize their spatial structure. Land multi-functionality studies have used land as a composite concept of society, economy and ecology and as a measure of the products and services that humans can obtain from using land. The current focus is on concepts and function identification, classification systems and function evaluations, and the main jumping off point is usually land use. Landscape multi-functionality and land multi-functionality tend to have some overlap in the research, with the focus on the ecosystem service functions, and landscape used as a tool for research on sustainable surface structures and spatial structures most often measured using the landscape pattern index ( $\mathrm{Wu}, 2013$; Zhou et al., 2019). Multi-functionality and overlapping functions have always been key issues plaguing functional classifications of production, living and ecological spaces (Shi and Zhang, 2018; Zou et al., 2018). Existing studies on production, living and ecological spaces include those on urban agglomerations, rural areas and river basins at various scales, such as countries, provinces, cities, counties, villages or towns, and with the primary focus on function identification, classification systems and function evaluations. 
Major function zoning in China aims to determine the role that each administrative unit should play in the national sustainable development system with comprehensive consideration given to their natural resource and environmental features, socio-economic development features and future development prospects. Rational organization of function zones is considered an important means of achieving orderly regional development (Fan et al., 2019). Major function zoning can be considered China's attempt to achieve a sustainable geographic and landscape pattern. In addition, it creates a new organizational unit of regional functions for managing territorial space, and its spatial organization has rich theoretical and academic connotations. Most previous research on major function zones has focused on scientific analysis, function zoning, monitoring and evaluation, performance appraisal, planning coordination and coordinated development, but the current tendency is toward research on supporting policies for major function zones (Liu et al., 2017; Wang and Fan, 2019a). Limited research on the spatial structure of major function zones has focused on spatial differentiation characteristics, such as population, sources of funding and construction land in provincial function zoning. Studies on spatial organizational rules and principles of major function zones of regions with proportional relationships between urbanization zones, agricultural development zones and ecological security zones as the basic parameters are extremely rare (Wang and Fan, 2019b). There are significant differences in the mechanisms driving regional function differentiation at different spatial scales, meaning that regional functions themselves have a scale effect, and the spatial structure of forms of spatial organization, specifically, poles, axes and surfaces, is also scaled. In other words, the spatial patterns of poles, axes and surfaces are different at different spatial scales, which means that major function zones exhibit significant scale characteristics. It is thus necessary to reveal the spatial structure characteristics of China's major function zoning at different scales.

This paper adopts a spatial structure perspective on China's major function zoning. It is based on modern regional function-structure theory, and uses the proportional relationship between urbanization zones, agricultural development zones and ecological security zones as its basic parameters. It uses GIS spatial statistics and analysis, spatial modeling and landscape morphology analysis to express quantitatively the agglomeration characteristics, spatial differences and topological structure of spatial structures at different levels, measuring the spatial gradient structure at the national scale, the pole-axis structure primarily at the provincial scale but with consideration given to the national scale, the core-periphery structure primarily at the provincial scale, and the spatial mosaic structure primarily at the county scale but with consideration given to the provincial scale, respectively. It also analyzes leading factors in areas such as natural geospatial differentiation, socio-economic spatial organization and regional spatial connections. Revealing the principles behind the spatial order of major function zones provides key parameters for spatial downscaling and accurate realization of major functions. It also provides an important reference for China's comprehensive geographical zoning, which has remained unresolved for some time.

\section{Method and data}

The formation of a regional function is the product of interactions in the spatial organization of physical geography and human activity, but there are significant differences in the mechanisms driving regional function differentiation at varying spatial scales. At the global 
and regional scales, land-sea differentiation, thermal zoning, land-sea fluctuations and continental shape patterns are the dominant factors in differentiating functions of terrestrial surface regions. Because integrated physical geography exhibits latitudinal zonality, longitudinal zonality, and sea-land vertical zonality spatial differentiations, it is highly likely that function zones will have zoned or regional spatial gradient characteristics. As the spatial scale narrows, geotectonic and geomorphic differentiation, provincial differentiation and segmental differentiation still play a leading role in the process of terrestrial surface function differentiation, but the role of zonal factors gradually decreases, while local factors and the role of the spatial organization of human activities gradually increases. In certain regional environments, especially in a nearly homogeneous natural geographic environment, the principles of socio-economic differentiation also play a leading role. Location theory tells us that the locations of socio-economic activities differ. Each region is suited to different degrees for different production and living activities, and urban systems, production systems and social systems all have potential optimal scales. Different scales also have potential optimal spatial organization structures for human activity. For example, the pole-axis structure explains the evolution process and formation mechanism of the spatial structure primarily expressed by pole and axis spatial morphology, and it describes the objective laws behind the occurrence and development of socio-economic spatial organization. Taking another example, the core-periphery structure characterizes the development status, existing problems and development potential of different regions. As such, the study of patterns of differentiation, driving mechanisms and interrelationships of regional functions all depend on the research scale, as changes in scale can cause relatively major changes in differentiation patterns and driving mechanisms. As the spatial scale changes, the relationship between function combinations, that is, the spatial structure, displays established changes.

Having analyzed the formation mechanisms of the aforementioned regional functions at different scales, we suppose that China's major function zoning has a spatial gradient structure at the national scale, a pole-axis structure primarily at the provincial scale but with consideration given to the national scale, a core-periphery structure primarily at the provincial scale, and a spatial mosaic structure primarily at the county scale but with consideration given to the provincial scale.

(1) The spatial gradient of major function zones means that the proportional relationship between urbanization zones, agricultural development zones and ecological security zones should display gradient differentiation in a specific direction or sequence. Using the area of function types and population as indicators, GIS buffer analysis is used to fit the corresponding indicators and corresponding degrees/zones (latitude and longitude, and distance from the coastline) or changing trends between regions with quantitative analysis (Figure 1).

(2) The pole-axis structure of major function zones is an integrated expression of the order of polar nuclei, the axes and surface function zones, including the optimal hierarchical system of polar nuclei, the optimal layout of axes and the optimal organization of the different function zones. The major function zone pole-axis structure is an orderly organization of pole-axis regional development systems and protective function zones. This characteristic is mainly reflected in the spatial differentiation of distance of different function zones to poles and axes. Using GIS spatial analysis and graphics, it is possible to identify function zone pole-axis structural elements at the national and provincial scales, to analyze axial 
scale effects at the national and provincial scales, and to measure the distance to polar nuclei and axes of different function zones.

(3) The core-periphery structure of major function zones refers to the principle of ring-like spatial differentiation, with distance from the core governing the proportional relationship between urbanization zones, agricultural development zones and ecological security zones. A multi-characteristic ellipse is the main method used, with a weighted center and central city (provincial capital) as the characteristic pole. The long-axis and short-axis distance and direction are characteristic lines, and the elliptical area, two-thirds of which is covered by urbanization zones, is the characteristic surface. The proportion and progression of urbanization zones, agricultural development zones and ecological security zones between different ellipses under the positive order of distance from the core position are the characteristic vector. The overall pattern and internal structure of the core-periphery structure is depicted by pole-axis-surface-vector comprehensive refinement.

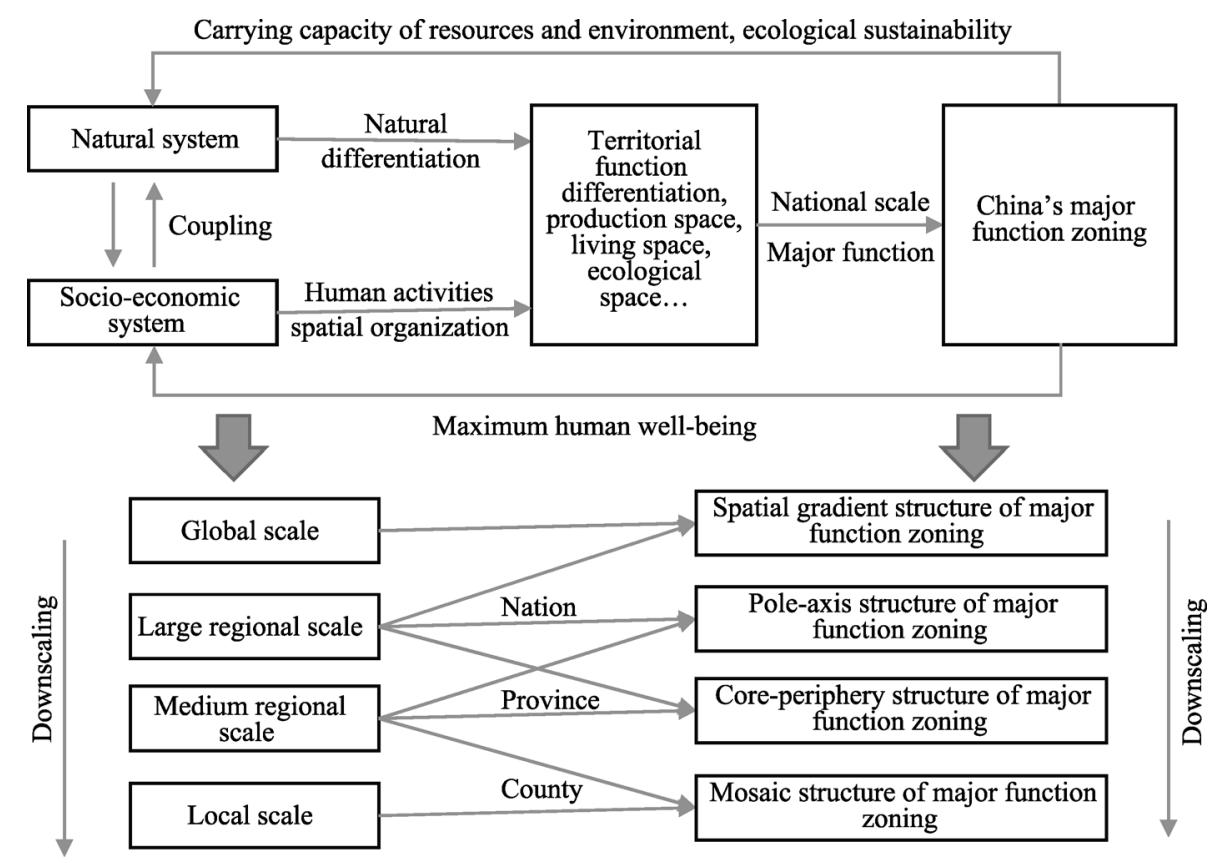

Figure 1 Approaches to multi-scale analysis of China's major function zoning

(4) The mosaic structure of major function zones refers to the interlocking distribution of the different function zone units that combine to make a whole, creating a spatial form of contiguous but separate pieces. These heterogenous units can be quantified using a variety of landscape indices, such as a dominance or fragmentation index. Major function zones display typical spatial heterogeneity, and there may be regular spatial correlations between different function zone units, which together make up the mosaic structure of major function zones. These spatial correlations include both topological analysis of different function zone combinations with common borders as well as proximity analysis of combinations of function zones in closest proximity.

Research data is based on Chinese major function zoning data, which was derived from the research paper titled "Draft of major function oriented zoning of China" by Fan Jie (2015). Major function zoning was a Chinese central government initiative to impose na- 
tional-level land use controls on its 9.6 million $\mathrm{km}^{2}$ of national territory (Figure 2). The program divides China's 2375 counties and cities into the following three major function zones. Urbanization zones account for a total area of 1.45 million $\mathrm{km}^{2}$ across 830 counties and districts throughout the country (calculated according to the area of county-level administrative districts, here and below). They are places with concentrations of human production and living activities, and they are the spatial agglomerations where large-scale industrialization and urbanization occur. Agricultural development zones account for a total area of 2.51 million $\mathrm{km}^{2}$ across 781 counties and districts, producing foodstuffs, ensuring food safety and maintaining the bottom line needed for sustainable human survival. Ecological security zones account for a total area of 5.66 million $\mathrm{km}^{2}$ across 764 counties and districts, providing ecological services, ensuring ecological security, and maintaining the bottom line of sustainability of China's natural environment. Heritage protection zones account for a total area of 1.18 million $\mathrm{km}^{2}$. The 8151 natural and cultural heritage protection zones are superimposed on the above three types of function zones. They are mainly sites of human culture and natural wealth that satisfy human cultural requirements. This study selects the first three types (urbanization zones, agricultural development zones and ecological security zones), which cover the whole country, and analyzes the area of function zones as a basic attribute, with reference to data on permanent resident population of counties in 2010.

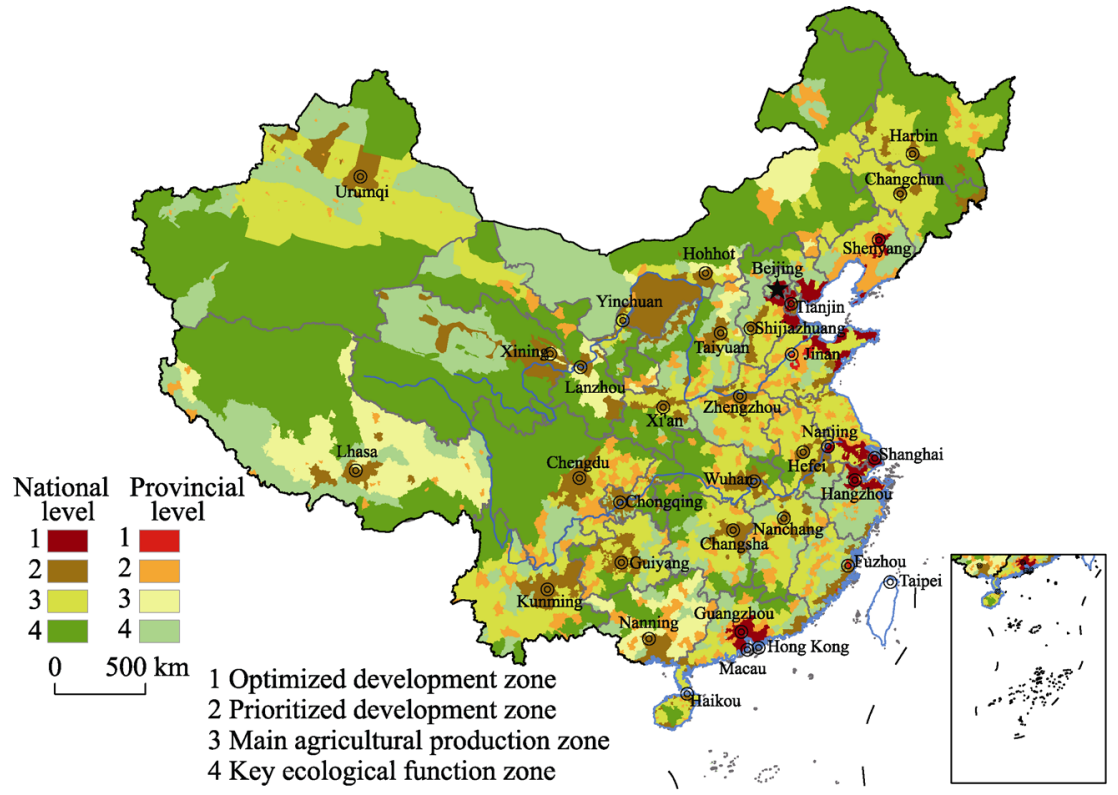

Figure 2 The draft of China's major function zoning (Fan, 2015)

\section{Results}

\subsection{Analysis of the spatial gradient structure at the national scale}

The research shows that major function zones have latitudinal, longitudinal, coastal distance and vertical distance spatial gradients. Specifically, urbanization zones are negatively correlated with latitude, distance from the coastline and vertical height, which means that the higher the latitude, the further away from the coastline, and the higher the altitude, the lower 
the proportion of urbanization zones. But they are positively correlated with longitude, so the higher the longitude, the higher the proportion of urbanization zones (see Figure 3). Ecological security zones have exactly the opposite correlations, and agricultural development zones show a range of differences. Specifically, the higher the longitude, the lower the latitude, and the lower the altitude, the higher the proportion of agricultural development zones. Agricultural development zones do not have obvious gradient characteristics when it comes to changes in distance from the coastline. In addition to displaying more significant regularity compared to land area proportions, the population indicator also shows the evolutionary characteristics of urban population movements toward high longitudes, low latitudes, coastal zones and low altitudes.
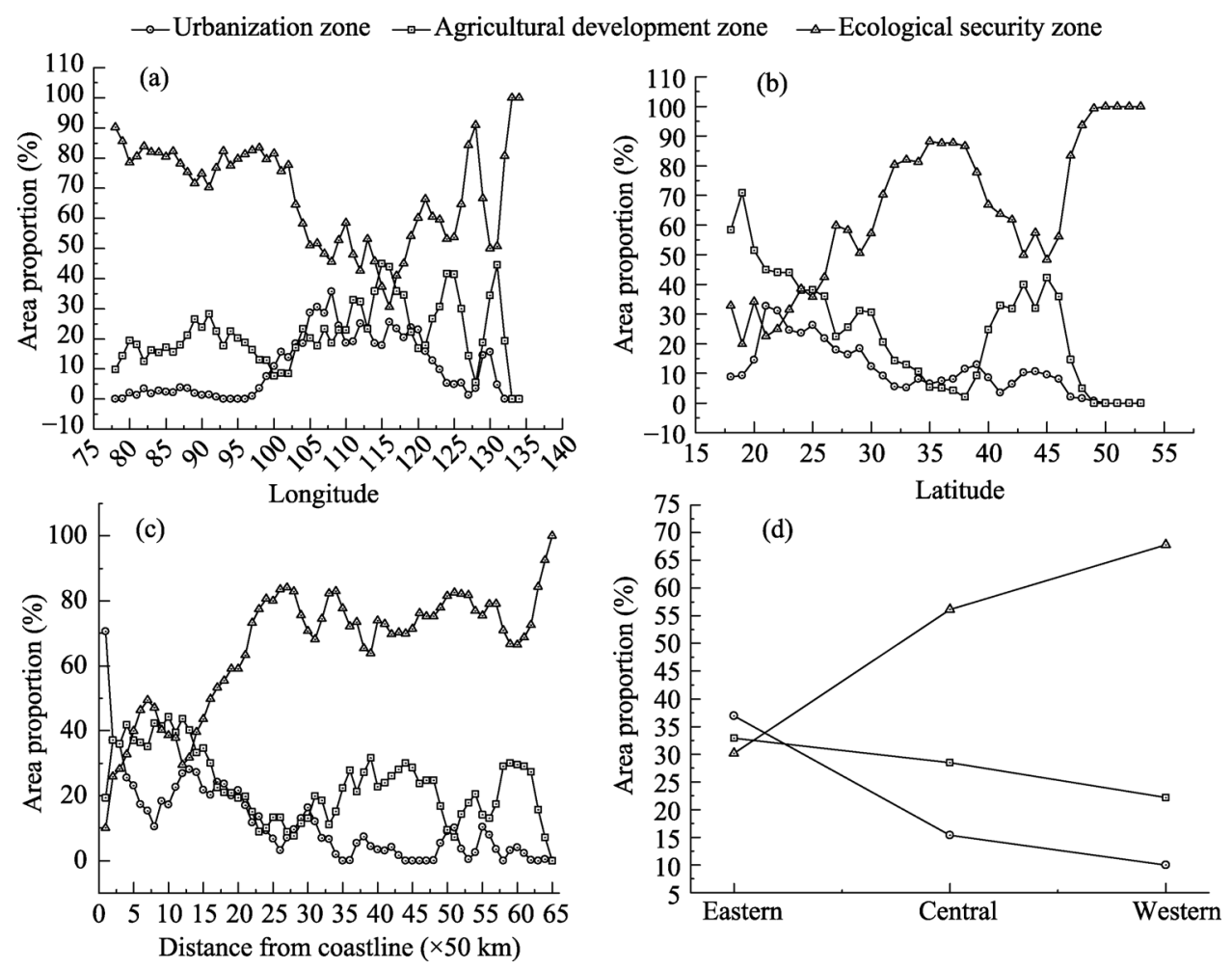

Figure 3 Area proportions of different function zones based on changes in latitude, longitude, distance from coastline and eastern-central-western China

Our research also shows provincial differentiation and eastern-central-western regional gradients. Provinces with higher proportions of urbanization zones are often those with the lowest proportions of ecological security zones. Of the 10 provinces with the lowest proportion of urbanization zones, seven ranked in the top 10 for the proportion of ecological security zones. Provinces with a high proportion of urbanization zones are often distributed along the coastline, while ecological security zones are distributed along the Da (Greater) and Xiao (Lesser) Hinggan Mountains, Taihang Mountains, Qinghai-Tibet Plateau and Yunnan-Guizhou Plateau. There are significant differences between coastal and inland areas. The distribution of ecological security zones is consistent with China's famous population boundary line, the $\mathrm{Hu}$ Line (an imaginary line proposed by geographer $\mathrm{Hu}$ Huanyong that divides China into two roughly equal parts), with provinces west of the line having a higher proportion of ecological security zones. The eastern-central-western gradients of urbaniza- 
tion zones, agricultural development zones and ecological security zones are 3.70:1.54:1, 1.48:1.28:1 and 1:1.86:2.25, respectively, with urbanization zones having the most significant eastern-central-western gradient. Further dividing the country into four major regions, it was found that there is an obvious western-northeastern-central-eastern spatial gradient, with the highest proportion of urbanization zones found in China's eastern region. Conversely, ecological security zones have an inverse gradient relationship, with a spatial gradient of $1: 1.25: 1.73: 2.28$.

\subsection{Analysis of the pole-axis structure primarily at the provincial scale but with consideration given to the national scale}

The results from a comprehensive evaluation of the polar nuclei show that different grades of polar nuclei exhibit significant spatial agglomeration and spatial differentiation characteristics (Figure 4). Polar nuclei with higher scores appear on the "two horizontal and three vertical" axes (of urbanization in China), especially where the coastal axis intersects the Yangtze River and Beijing-Guangzhou axes. By identifying the area, population and economic weighted main axes of provinces' development using the distribution characteristics of polar nuclei, it was found that three-quarters of axes in China are longitudinal axes, at $45^{\circ}-135^{\circ}$, mainly in the coastal and central provinces (see Figure 4). Because the development axes of provinces in western China have topographical constraints, such as often being along large mountain ranges, they are mainly horizontal axes. The majority of the area, population and economy weighted main axes of provinces' major function zones basically coincide, with nearly $85 \%$ of provinces having less than $5^{\circ}$ difference in the direction of their axes.

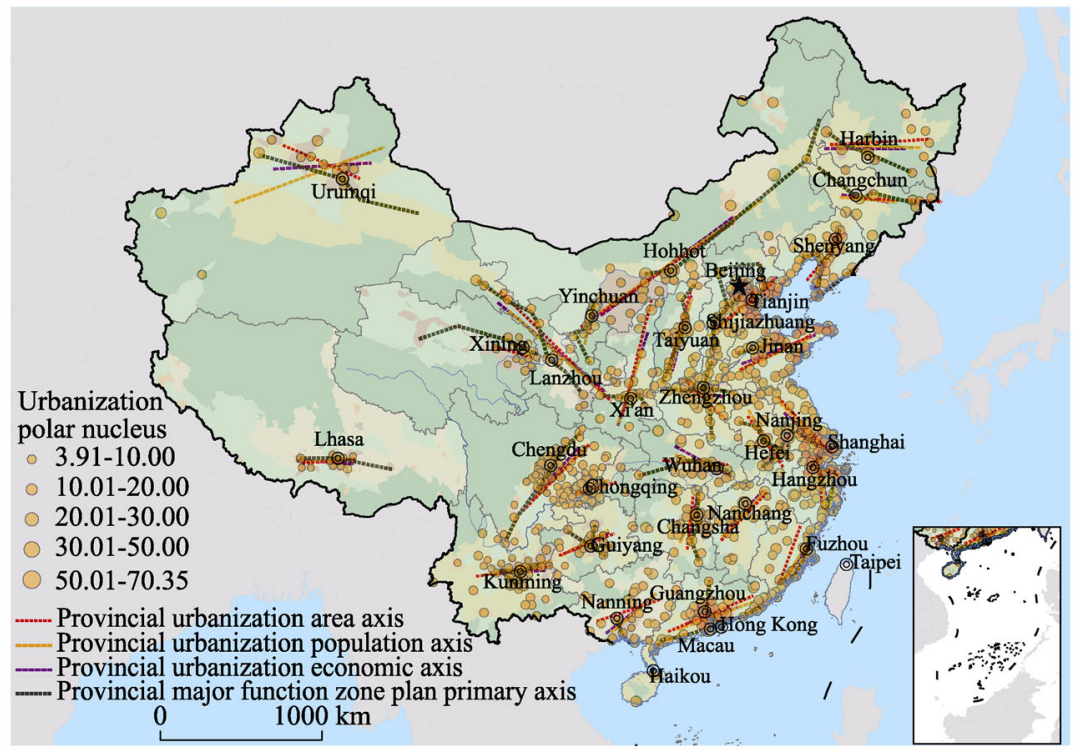

Figure 4 Elements of provincial polar-axis structure of major function zoning

Looking at the spatial distribution of different function zones, urbanization zones are concentrated on provincial axes close to agricultural development zones, while ecological security zones are often located in peripheral areas. It can be said that in the course of developing the major function zone pole-axis regional system, consideration was given to the 
convenience of supplying agricultural products as well as the barrier function of ecological security zones. Further research on spatial differentiation of proportion and distance of function zones from the first order axis of each province showed that as distance from the axis increases, the proportion of urbanization zones decreases, and the proportion of ecological security zones increases. This is a common feature in all provinces.

By matching the function zone identification axes with provincial major function zone program axes, it was found that the degree of matching between identification axes in the western region is better than in the central and coastal regions, and the population axes in the coastal region are closer to the planned axes. This is inconsistent. Characteristic lines are mainly affected by minor axes, including in Xinjiang, Heilongjiang, Guizhou, Henan and other provinces in the central and western regions. The axes of the central and western regions are clearly limited by topography, often along large mountain ranges, in an east-west direction. The eastern plains are largely dominated by north-south axes, and socio-economic spatial linkage plays a key role. Looking at the provincial axes and national horizontal and vertical axes, it can be seen that vertical axes are more consistent than horizontal axes. The highest in order are the Baotou-Kunming rail route, coastal, and Beijing-Harbin and Beijing-Guangzhou axes, of which, the Baotou-Kunming route has 100\% coincidence. In inland areas, especially in the central and western regions, axes are more consistent than near the coast, and the coincidence is higher than $80 \%$; whereas, the coastal region has coincidence of only $37 \%$. This is because north-south connections have always been the main links in China, facilitating the movement of northern coal to the south and southern grain to the north. In addition, China's terrain rises from the coast to the inland, so transportation routes perpendicular to the coast are difficult to construct. Construction of north-south routes better suits China's natural conditions.

\subsection{Analysis of the core-periphery structure primarily at the provincial scale}

The core-periphery structure exists in all China's provinces, as the proportional relationship between urbanization zones, agricultural development zones and ecological security zones and distance to the core displays ring-like spatial differentiation (Figure 5). When the spatial distribution of urbanization zones conforms to normal distribution, the proportion of urbanization zones is the highest at the center and decreases toward the periphery, but the position of the core and the periphery, the function attributes of the periphery, and the proportions of and distances between different function zones of the core and the periphery, vary by province. The core of each province is invariably located near the provincial capital. The distance from the core to the provincial capital is generally longer in coastal provinces and shorter in provinces of the central and western regions. The central and western regions mainly have single cores, while provinces of the coastal region tend to have multiple cores and a more balanced core-periphery structure. The movement of peripheries accords with boundaries of major terrain. Almost all ecological security zones have been established in peripheral areas. This is common across the country, but the proportions of ecological security zones and urbanization zones differ in different provinces. Further analysis of the positional relationship between different function zones shows that the degree of overlap between urbanization zones and agricultural development zones is above $60 \%$ in more than two-thirds of provinces; the core and periphery of urbanization zones are in the inner ring, 
and agricultural development zones are located outside urbanization zones. The overlap between urbanization zones and ecological security zones, on the other hand, is above $70 \%$ in 10 provinces, and the overlap between agricultural development zones and ecological security zones is above $60 \%$ in more than half of provinces, with ecological security zones generally located in peripheral areas.

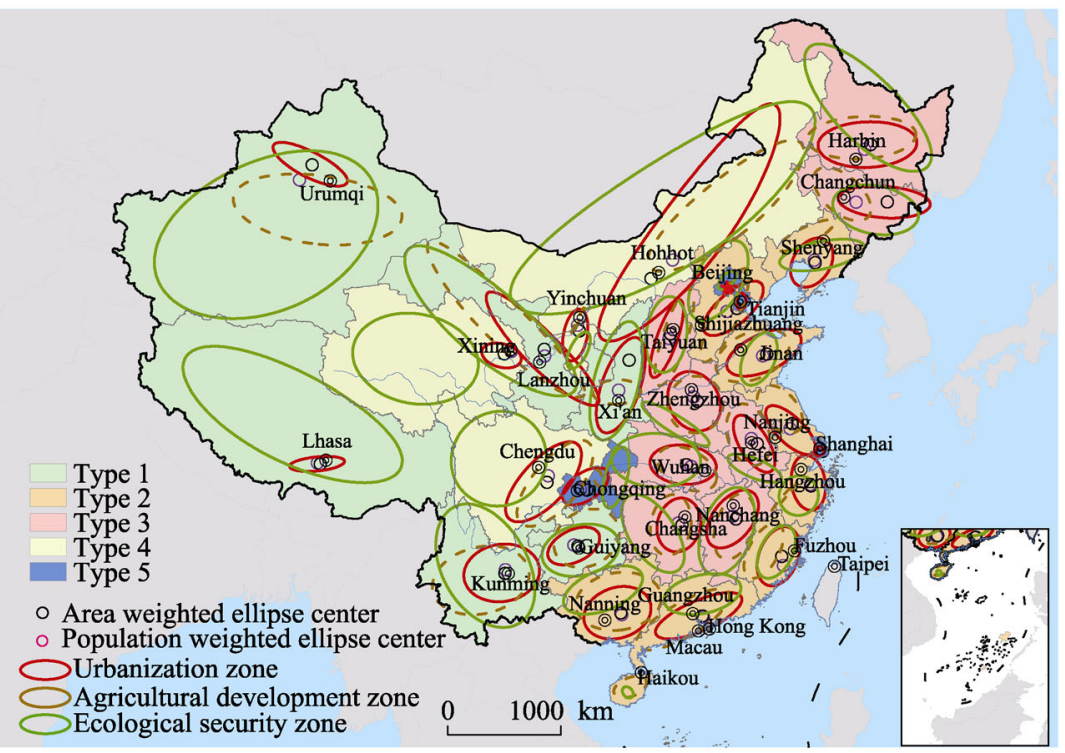

Figure 5 Provincial core-periphery characteristics of China's major function zoning

Generally speaking, it is evident that the core-periphery structure of major function zones has the following main features: The majority of provinces have a core-periphery model with urbanization as the inner ring, agricultural development as the middle ring, and ecological security as the outer ring. Provinces with this model include Xinjiang, Gansu, Tibet, Yunnan, Guizhou and Shaanxi. Provinces with a core-periphery model consisting of urbanization zones at the coast and agricultural development or ecological security zones inland include Liaoning, Hebei, Shandong, Jiangsu, Zhejiang, Fujian, Guangdong and Guangxi. Typical provinces with a core-periphery model consisting of urbanization zones in the inner ring and agricultural development zones at the periphery include the central provinces of Jiangxi, Shanxi, Jilin, Heilongjiang, Anhui, Henan, Hunan and Hubei. The provinces of Qinghai, Ningxia, Sichuan and Inner Mongolia have a core-periphery structure in which their urbanization zones are close to provincial borders and contiguous with other provinces, while their ecological security zones extend internally. The four municipalities directly under the central government, namely, Beijing, Tianjin, Shanghai and Chongqing, have classic core-periphery structures, with urbanization zones at their core. The core-periphery structure is based on the natural geographic environment. Specifically, water resources determine the location of urbanization zones, that is, the location of the core. Ecological encroachment determines the spatial distribution of ecological security zones, that is, the periphery. The weaker an area's physical geographic constraints, the stronger the impact socio-economic activities will have. The pole-axis and core-periphery structures are not only constrained by the natural geographic environment and the spatial organization of human social activities, 
but also by the spatial connections between different regions. This is most prominent on more developed plains, where accessibility to the provincial capital often determines the direction of the axis. The stronger the spatial connections, the larger the scope of the core area, and the more the gap between the core and periphery is mitigated.

\subsection{Analysis of the spatial mosaic structure primarily at the county scale but with consideration given to the provincial scale}

The spatial mosaic structure is the most basic and most intuitive spatial structure of China's major function zoning. This spatial mosaic structure has both features of spatial heterogeneity, such as agglomeration and dispersion, as well as the features of mutual adjacent topological correlation and spatial proximity. Analysis of spatial heterogeneity shows that prefecture-level cities dominated by urbanization are mainly located along China's "two horizontal and three vertical" urbanization axes, and the continuity of the vertical axes is higher than the horizontal axes (Figure 6). It also shows that the prefecture-level cities dominated by ecological security have as their skeleton and outline China's main mountain ranges. Areas dominated by ecological security are often areas with the best connectivity with other ecological security areas and less fragmentation with urbanized areas; whereas, areas dominated by agricultural development tend to have higher fragmentation and poor connectivity. Differences in regions dominated by urbanization are quite stark. Eastern coastal areas that are relatively developed and have relatively balanced development are most contiguous and have higher fragmentation; the central plains have poor contiguousness and higher fragmentation; the mid-western region is relatively compact, has good contiguousness and has a low degree of fragmentation; and the mid-western areas besides the mountains often have low contiguousness and a high degree of fragmentation.
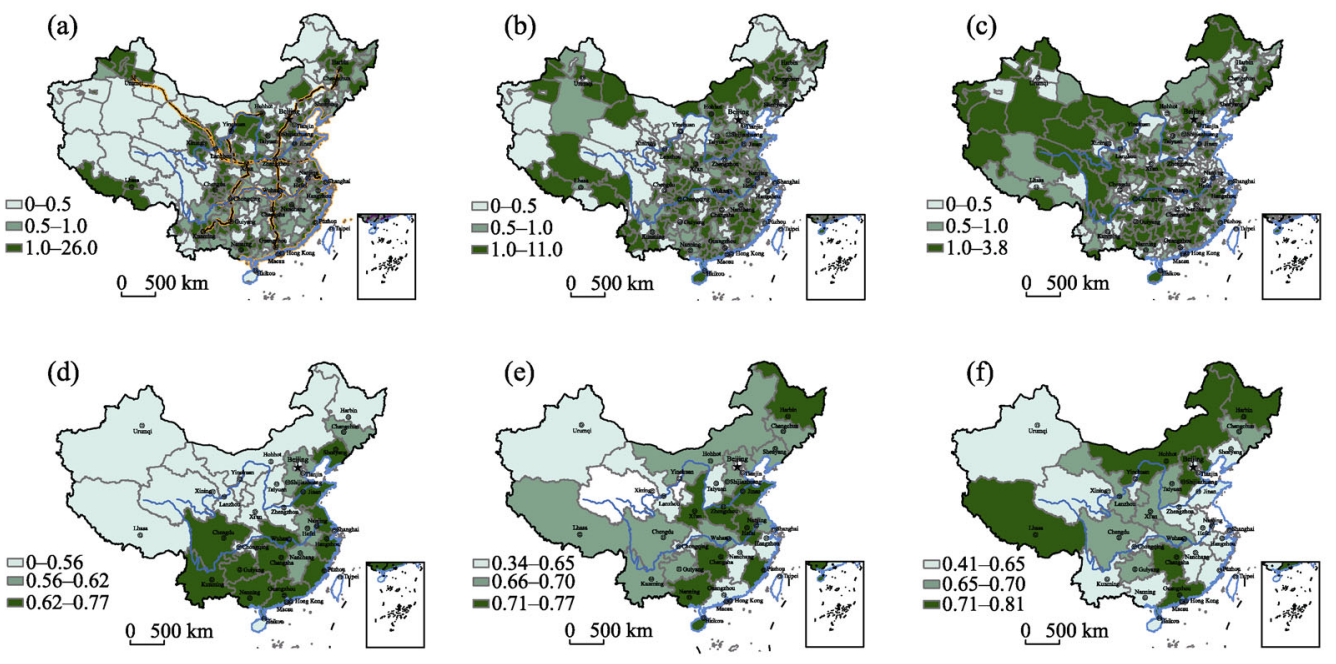

Figure 6 Analysis of spatial heterogeneity of major function zoning

A spatial matrix is used to analyze topological features of the common borders of different types of function zones, analyze the numbers of other urbanization zones, agricultural development zones and ecological security zones around urbanization zones, and work out the distribution of these types of values (Figure 7). This is then used to characterize the probability of combinations of different function zones. Our research shows the following: 


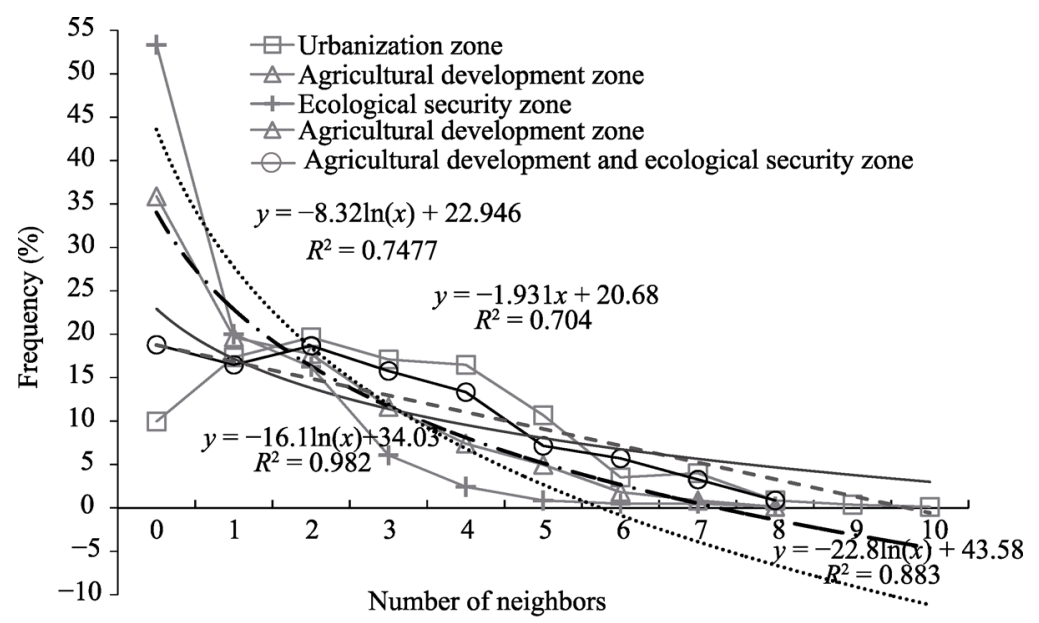

(a) Topological analysis of common boundaries

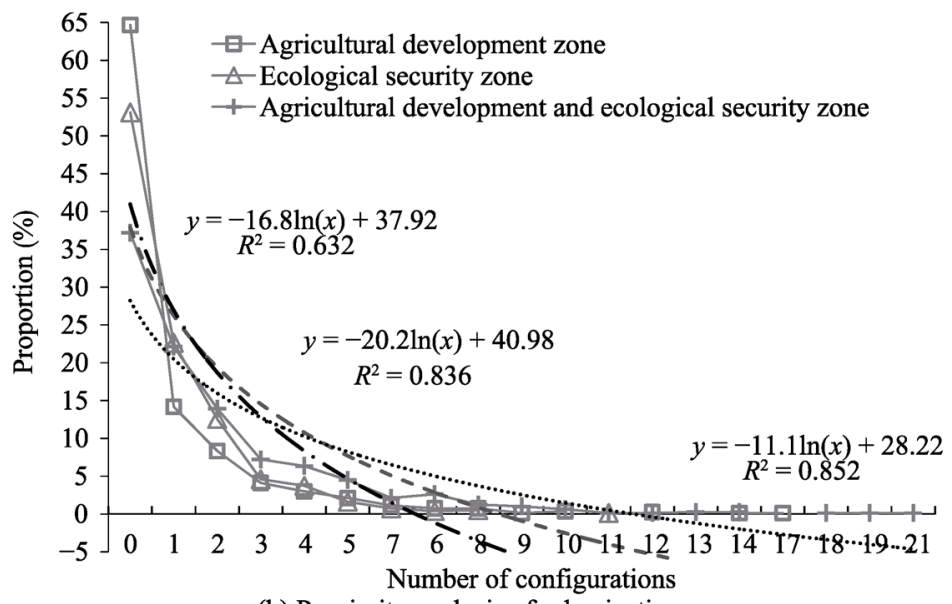

(b) Proximity analysis of urbanization zones

Figure 7 Spatial correlation analysis of different types of function zones

First, urbanization zones are largely clustered together, with $53.09 \%$ of zones having three or more other urbanization zones next to them, and $18.79 \%$ of urbanization zones having only urbanization zones next to them, which is a vital foundation for creating urban agglomerations and they are the main areas for balancing the core-periphery structure. Second, $64.14 \%$ of urbanization zones have one or more agricultural development zones next to them, indicating that most urbanization zones have relatively good supplies of labor and food. As the number of neighboring zones increases, the curve shows a logarithmic decline. A further observation is that $15.17 \%$ of urbanization zones are next to three or more agricultural development zones. This is an important basis of the pronucleus structure or pole-axis structure. Third, $46.67 \%$ of urbanization zones have one or more ecological security zones next to them; of which, $42.30 \%$ have between one and three. Looking at combinations of urbanization zones and agricultural development zones or ecological security zones, we discover that as the number of combinations increases, the proportion of urbanized areas displays a typical logarithmic function relationship. As many as $81.21 \%$ of urbanization zones have more than one 
agricultural development or ecological security zone next to them. Agglomeration and diffusion between different regions have always played important roles in spatial mosaic formation, which is manifested as the core region organizing the creation of the periphery and new core regions through the supply, market and administrative systems. It is through the action of agglomeration and diffusion that different function zones form various spatial combinations, resulting in the spatial mosaic structure.

\section{Conclusions and discussion}

Based on China's major function zoning, this study set out to explore the development mechanisms of regional functions at different scales using GIS spatial statistics and analysis, spatial modeling, and landscape morphology analysis in order to measure the spatial gradient structure at the national scale, the pole-axis structure primarily at the provincial scale but with consideration given to the national scale, the core-periphery structure primarily at the provincial scale, and the spatial mosaic structure primarily at the county scale but with consideration given to the provincial scale, respectively. It also analyzed the leading factors of all types of spatial structure in terms of natural geospatial differentiation, socio-economic spatial organization and regional spatial connections.

(1) Major function zones have a spatial gradient structure, which is prominently represented by latitudinal and longitudinal gradients, a coastal distance gradient, a vertical distance gradient, and an eastern-central-western regional gradient. The proportion of urbanization zones is negatively correlated with latitude, distance from the coastline and vertical height, and it is positively correlated with longitude; whereas, the proportion of ecological security zones has exactly the opposite correlations. We also discovered provincial differentiation and eastern-central-western regional gradients. Provinces with higher proportions of urbanization zones are often those with the lowest proportions of ecological security zones.

(2) The core-periphery structure is prevalent in all provinces of the country. This means that the proportional relationship between urbanization zones, agricultural development zones and ecological security zones displays a ring-shaped spatial differentiation principle with distance from the core, but there are differences in terms of the location of the core, axis orientation, periphery function attributes, proportions of internal functions, and so on. Provinces in China's western region have clear ring-like differentiation of urbanization, agricultural development and ecological security zones, and almost all ecological security zones are in peripheral areas. In the provinces of China's central region, the proportion of agricultural development zones in peripheral areas has increased. Coastal provinces commonly have multiple cores, which are dominated by urbanization zones.

(3) At the national and provincial scales, a pole-axis structure exists. Urbanization zones are a collection of polar nuclei at different levels. The level and spatial distribution of the polar nuclei determine the development axis. The general principle of the pole-axis structure is that as one moves along the distance axis, the proportion of urbanization zones decreases and the proportion of ecological security zones increases. Moreover, the higher the axis level, the higher the proportion of urbanization zones within the initial measurement distance and the lower the proportion of ecological security zones and the faster the decrease in the proportion of urbanization zones within the same distance.

(4) The city and county scale displays a spatial mosaic structure. This structure has both features of spatial heterogeneity, such as agglomeration or dispersion, as well as features of 
mutual, adjacent topological correlation and features of spatial proximity. Differences within spatial heterogeneity features of the spatial mosaic structure are considerable, not only in terms of scale, but also in terms of types of zones. The organization of different function zones has potential optimal scales under the two forces of agglomeration and dispersion. Mutual combinations of urbanization zones constitute an important basis of urban agglomerations. Contiguous and fragmented agricultural development zones reflect the self-requirement of agricultural production to be linked and the result of developing urbanization. Under the precondition of observing the objective principles of natural ecosystems, ecological security zones constitute a network of ecological barriers across the country and for urban areas (and agglomerations).

The following are the results of analyzing the spatial structure of major function zones from the three dimensions of natural geospatial differentiation, socio-economic spatial organization and regional spatial connections: At the national scale, the natural geographic environment dominates, with latitudinal zone gradients dominated by heat zones, longitudinal and coastal distance gradients dominated by arid and wet zones, and vertical zonality and east-center-west regional gradients dominated by geotectonic topography. At the provincial scale, the natural geographic environment is a basic constraint, with the influence of socio-economic development gradually emerging. Regions with weaker natural geographic environment constraints are more significantly influenced by socio-economic development. For example, the pole-axis and core-periphery structures in provinces in China's western region are greatly influenced by major geographical boundaries, and the pole-axis and core-periphery structures in provinces in China's eastern region are influenced by existing urban systems, socio-economic layouts and methods of socio-economic organization. At the mesoscale, the overlapping effects of the natural environment, society and economy, and interregional interactions are increasingly apparent, the natural geographic environment has objective regional differences, and there are differences in terms of the spatial orientation of economic and social activities and the strength of connections between regions. In addition, ecosystems, production systems and social systems all have potential optimal scales. These are the main influencing factors of the spatial structure of major function zones. In addition, there is an interactive relationship between types of spatial structure, which manifests as a higher-level spatial structure becoming the background influence on the structure at the next lower level, and the features of the lower-level structure reshaping the structure at the next higher level. The mosaic structure is the result of the combined effects of gradient, pole-axis and core-periphery.

There are still some limitations to the research in this study, which require further exploration: First, China's major function zoning is a vision of the future, and the major function pole-axis structure itself is final, static and ideal. The spatial structure characteristics of the various function zones, such as population and economy, are constantly changing. Due to the fact that demand structure, subjective cognition and production technology of society are constantly changing, the functions assigned to a region may differ at different stages of development. These factors are driving continuous changes in major functions and their spatial structure. This requires further exploration. Second, when expounding the interactions and the coupling mechanisms between pole, axis and surface function zones in this study, little attention was given to the interactive relationship between agricultural development zones, ecological security zones and the poles and axes. In future, this could be elaborated using relevant theories from economics and ecology as well as other more technical means. Finally, 
future studies should focus on micro-analysis of industries, using economic theory to explain spatial competitive relationships and comparative advantages as well as to explain further the role of diffusive forces in the formation and evolution of the spatial structures of major function zones. Further research on spatial connections is needed, particularly on the role of interregional flows of information, capital and talent in regional connections, and studies should explore the role of fluid spaces in reshaping the spatial structure of major function zones as well as the causes and mechanisms of topological relationships between different function zones.

\section{References}

Baerwald T J, Firth P L, Ruth S L, 2016. The dynamics of coupled natural and human systems program at the US National Science Foundation: Lessons learned in interdisciplinary funding program development and management. Current Opinion in Environmental Sustainability, 19: 123-133.

Esmail M, Mahmod W E, Fath H, 2019. Assessment and prediction of shoreline change using multi-temporal satellite images and statistics: Case study of Damietta coast, Egypt. Applied Ocean Research, 82: $274-282$.

Fan J, 2015. Draft of major function oriented zoning of China. Acta Geographica Sinica, 70(2): 186-201. (in Chinese)

Fan J, Li P X, 2009. The scientific foundation of major function oriented zoning in China. Journal of Geographical Sciences, 19(5): 515-531.

Fan J, Wang Q, Wang Y F et al., 2018. Assessment of coastal development policy based on simulating sustainable land-use scenario for Liaoning Coastal Zone in China. Land Degradation \& Development, 29(8): 2390-2402.

Fan J, Wang Y F, Ouyang Z Y et al., 2017. Risk forewarning of regional development sustainability based on a natural resources and environmental carrying index in China. Earth's Future, 5(2): 196-213.

Fan J, Wang Y F, Wang C S et al., 2019. Reshaping the sustainable geographical pattern: A major function zoning model and its applications in China. Earth's Future, 7(1): 25-42.

Fu B J, 2017. Geography: From knowledge, science to decision making support. Acta Geographica Sinica, 72(11): 1923-1932. (in Chinese)

Kates R W, 2011. What kind of a science is sustainability science? Proceedings of the National Academy of Sciences of the United States of America, 108(49): 19449-19450.

Li Q Q, Lu Y, Hu S B et al., 2016. Review of remotely sensed geo-environmental monitoring of coastal zones. Journal of Remote Sensing, 20(5): 1216-1229.

Liu J G, Dietz T, Carpenter S R et al., 2007. Complexity of coupled human and natural systems. Science, 317(5844): 1513-1516.

Liu W C, Liu J Y, Kuang W H et al., 2017. Examining the influence of the implementation of Major Function-oriented Zones on built-up area expansion in China. Journal of Geographical Sciences, 27(6): 643-660.

Loorbach D, Frantzeskaki N, Avelino F, 2017. Sustainability transitions research: Transforming science and practice for societal change. Annual Review of Environment and Resources, 42: 599-626.

Qiao K, Zhu W Q, Hu D Y et al., 2018. Examining the distribution and dynamics of impervious surface in different function zones in Beijing. Journal of Geographical Sciences, 28(5): 669-684.

Russell R, Guerry A D, Balvanera P et al., 2013. Humans and nature: How knowing and experiencing nature affect well-being. Annual Review of Environment and Resources, 38: 473-502.

Shi Z Q, Zhang S Y, 2018. Spatio-temporal pattern changes of land space in Hengduan Mountains during 1990-2015. Journal of Geographical Sciences, 28(4): 529-542.

Wang Y F, Fan J, 2019a. Analysis of the core-periphery structure of Major Function Zones in China. Acta Geographica Sinica, 74(4): 710-722. (in Chinese)

Wang Y F, Fan J, 2019b. Spatial analysis of national-provincial pole-axis structure based on major function zoning in China. Geographical Research, 38(7): 1651-1663. (in Chinese)

Wu J G, 2013. Landscape sustainability science: Ecosystem services and human well-being in changing landscapes. Landscape Ecology, 28(6): 999-1023.

Zhou B B, Wu J, Anderies J M, 2019. Sustainable landscapes and landscape sustainability: A tale of two concepts. Landscape and Urban Planning, 189: 274-284.

Zou L L, Wang J Y, Hu X D, 2018. An classification systems of production-living-ecological land on the county level: Theory building and empirical research. China Land Science, 32(4): 59-66. (in Chinese) 